\title{
Tuberculosis Multimorbidity: A Global Health Challenge Demanding Urgent Attention
}

Author 1. Kamran Siddiqi (corresponding author)

Affiliation: Department of Health Sciences, The University of York and Hull York Medical School

Address: Seebohm Rowntree Building

Department of Health Sciences

The University of York

Heslington, YO10 5DD

Author 2. Brendon Stubbs

Affiliation: Institute of Psychiatry, Psychology \& Neuroscience, King's College London,

Author 3. Yan Lin

Affiliation: International Union Against Tuberculosis and Lung Disease

Author 4. Helen Elsey

Affiliation: Department of Health Sciences, The University of York and Hull York Medical School

Author 5. Najma Siddiqi

Affiliation: Department of Health Sciences, The University of York and Hull York Medical School

Key Words: Tuberculosis, Multimorbidity, comorbidity, non communicable diseases

Word Count: 1,527 excluding the table, acknowledgements and references 


\section{Tuberculosis Multimorbidity: A Global Health Challenge Demanding Urgent Attention}

Multimorbidity, defined by the World Health Organisation's (WHO) ${ }^{1}$ as the existence of two or more chronic health conditions in the same individual, is associated with adverse health outcomes, ${ }^{2}$ and increased healthcare costs. Tuberculosis (TB) commonly coexists with a range of other serious chronic conditions such as HIV, diabetes and depression. ${ }^{3}$ However, within health systems with limited capacity and access to care, services (including TB services) typically focus on single conditions and neglect to identify and manage multimorbidity, missing opportunities to prevent, screen and treat coexisting chronic conditions.

Whilst there has been some progress in our understanding of TB coexisting with single other conditions (e.g. TB in people living with HIV), ${ }^{4}$ little is known about the burden, characteristics and clusters of multimorbidity in people with TB - referred to hereafter as TB multimorbidity. We wish to highlight the challenge posed by TB multimorbidity and its current neglect in healthcare. The editorial invites policy makers and providers to rethink more joined-up, patient-centred care for TB that capitalises on opportunities and synergies in the approach to recognise and manage TB multimorbidity.

\section{The burden and characteristics of TB multimorbidity}

The magnitude of the mortality and morbidity burden associated with TB is well recognised and is reflected in the global advocacy efforts, financial investments and technical advances in the prevention and control of TB. ${ }^{5}$ However, despite its undoubted importance, evidence on the determinants, disease patterns, burden and consequences of TB multimorbidity is only recently emerging. TB multimorbidity is associated with complex mental and physical symptoms, ${ }^{6}$ worsening of disabilities, ${ }^{7}$ poor quality of life, ${ }^{8}$ excess healthcare use ${ }^{910}$ premature deaths, ${ }^{11}$ and high healthcare costs. ${ }^{12}$

Among mental health conditions, depression affects one in every four individuals with TB, adversely affecting adherence to TB treatment and recovery. ${ }^{13,14}$ TB and depression share several common determinants and risk factors; ${ }^{15,16}$ depression also affects the immune system $^{17}$ and increases the risk of acquiring TB. ${ }^{16}$ If it remains untreated, depression in people with TB may lead to treatment default, greater disability, poorer quality of life and increased risk of death. ${ }^{18}$ Conversely, TB may increase the severity of depression by 
adversely affecting quality of life ${ }^{19}$ and because of the additional stigma associated with TB. ${ }^{20}$

Among chronic infections, the evidence on the co-occurrence and the bidirectional adverse interactions between TB and HIV is most well established. HIV increases the risk of acquiring TB 20- to $40-$ fold; ${ }^{21}$ hence the HIVIAIDS epidemic between 1990-2005 led to a five-fold increase in TB incidence in sub-Saharan Africa. ${ }^{12}$ Currently, an estimated one in six people with TB has HIV co-infection; the presence of TB also worsens HIV outcomes. ${ }^{22}$ For example, the TB mortality rate in those with HIV co-infection may be twice that of those without HIV. ${ }^{4}$

Most adults with TB have one or more co-existing non-communicable diseases (NCDs), the likelihood increasing with age..$^{23}$ In a secondary data analysis of the World Health Survey (WHS) conducted in 48 low- and middle-income countries (LMICs), we found that around two-thirds of people with TB had at least one NCD. ${ }^{23}$ Prevalence of NCD in people with TB was twice that of those without TB. ${ }^{23}$ This clustering of TB with NCDs is attributable to several common risk factors, including social factors (stigma), ${ }^{20}$ economics (poverty), health risk behaviours (smoking, alcohol and drug abuse), impaired immunity and drug interactions. ${ }^{21}$

TB and NCDs interact adversely, resulting in a multiplier effect on their respective disease burdens and health outcomes. ${ }^{24}$ The WHS analysis estimated at least one-third of all morbidity (measured as Years Lived with Disability [YLD]) in individuals with TB is attributable to coexisting NCDs. ${ }^{23}$ At a population level, TB and NCDs act as a syndemic or synergistic epidemic, which sustains and magnifies both epidemics. NCDs and their risk factors have a substantial influence on maintaining the TB epidemic in high-burden countries; 24

Diabetes, ${ }^{25}$ and chronic respiratory disorders, ${ }^{26}$ are considered to be the two most common NCDs coexisting with TB. Diabetes increases the risk of developing active TB three-fold; in high-TB and high-diabetes burden countries such as India, almost every other person with TB may have diabetes or pre-diabetes. ${ }^{27}$ People with both TB and diabetes are likely to have poor outcomes: they are less likely to recover from TB and more likely to die, ${ }^{28}$ suffer TB recurrence, ${ }^{28}$ have a poor quality of life ${ }^{29}$ and develop multidrug resistant TB. ${ }^{30}$ Moreover, TB adversely affects glycaemic control and its progression towards complications. ${ }^{31-33}$ The 
global diabetes epidemic impedes achievement of Global End TB Targets. ${ }^{5}$ And poor TB outcomes increase the global disease burden attributable to diabetes through poor glycaemic control and rapid progression to complications. ${ }^{21}$

TB also increases the risk of developing and worsening outcomes of chronic respiratory disorders. ${ }^{34}$ Those with both chronic respiratory disorders and active TB have a two-fold increased risk of death within 12-months of being diagnosed with TB. ${ }^{35}$ TB also increases the risk of developing chronic respiratory infections during and after anti-TB treatment. ${ }^{36,37}$

\section{Complex TB multimorbidity}

In addition to the increased prevalence of comorbid individual chronic conditions in TB, there is also the significant problem of "complex" TB multimorbidity, which poses significant additional challenges for people with TB and TB services. We define complex TB multimorbidity as the co-occurrence of two or more chronic conditions with TB. There is an urgent need to better understand what are the most common 'clusters' of mental disorders, chronic communicable and non-communicable diseases (NCD) that co-occur with TB, which clusters are associated with greatest burden, and how to address them. ${ }^{38}$

\section{The neglect of multimorbidity in TB healthcare}

The needs of people with multimorbidity, including TB multimorbidity, are largely neglected in traditional, typically single-disease focused health programmes, ${ }^{6}$ despite the fact that the common patient experience is of multiple chronic conditions. ${ }^{38}$ The care for people with TB multimorbidity typically remains vertical, fragmented and non-personalised, with numerous missed opportunities to prevent, screen and manage coexisting serious chronic conditions. On the other hand, the synergies between TB and other chronic conditions (and between these other conditions) demand effective joined-up approaches to prevention, screening and treatment of co-occurring conditions. The significant disease burden attributable to multimorbidity and the potential health gains through exploiting synergies in managing multiple chronic conditions, makes TB multimorbidity a priority in high burden countries. 


\section{The opportunity to address coexisting chronic conditions}

The need to address multimorbidity through effective, integrated and scalable interventions in healthcare is gaining international attention. ${ }^{39} \mathrm{WHO}$ is advocating for an integrated person-centred approach through coordination and alignment within and across healthcare settings. ${ }^{40}$ Moreover, there is a growing recognition of the need for, and strong global and national policy support to address TB multimorbidity (e.g. WHO's 'End TB Strategy,' Stop TB Partnership's 'The Paradigm Shift: Global Plan to End TB 2018-2022' and the 'Global Fund Support for Co-infections and Co-morbidities'). ${ }^{41}$ An increasing global focus on NCDs and the establishment of NCD services in several high TB burden countries is also an opportunity to coordinate care across NCDs and TB services to address multimorbidity.

TB services may be particularly well-placed to develop innovative approaches to address multimorbidity. Services endeavour to engage with those diagnosed with TB for at least six months, offering multiple opportunities to prevent, screen and manage other chronic conditions. Their (relatively) strong and well-resourced infrastructure may provide a platform for interventions to address multimorbidity, without sacrificing recent gains in TB outcomes. ${ }^{5}$ The concordance between treatments for TB and for some NCDs is also an opportunity to link care across healthcare teams to address TB multimorbidity. ${ }^{12}$

There do exist evidence-based frameworks for integration ${ }^{42}$ and person-centred care,${ }^{40}$ screening approaches, ${ }^{43,44}$ and effective interventions, ${ }^{45,46,47}$ that can be used to tackle TB multimorbidity. In people with HIV-associated TB, antiretroviral therapy (ART) has been hugely beneficial in reducing mortality and recurrence of TB. ${ }^{48}$ Cost-effective interventions to address common risk factors for TB and NCDs are rarely offered routinely within TB healthcare, ${ }^{49}$ yet could prevent the onset and/or progression of multimorbidity. For example, there is emerging evidence that smoking cessation support is effective ${ }^{49}$ and improves TB outcomes. ${ }^{50}$ Similarly, better diabetes control in TB patients through screening, treatment and monitoring could improve TB treatment success, reduce TB transmission and potentially avert more than a million deaths in high-burden countries over the next 15 years. ${ }^{51}$ Likewise, screening for and treating depression would not only reduce its severity but also improve motivation and adherence to TB treatments. ${ }^{16}$ Table 1 outlines the interventions for screening, prevention and management of TB multimorbidity that could be implemented in a resource-limited high TB burden country. Patients will need continued care for their chronic conditions even after completing their anti-TB treatment. The proposed interventions to 
address TB multimorbidity will also require additional financial support from domestic and international sources e.g. Global Fund.

Table 1: Interventions for screening, prevention and management of TB multimorbidity

\begin{tabular}{|l|l|l|}
\hline HIV and AIDS & $\begin{array}{l}\text { Screening } \\
\text { HIV testing and } \\
\text { counselling for patients } \\
\text { diagnosed TB }\end{array}$ & $\begin{array}{l}\text { Initiating ART in people living with HIV, } \\
\text { regardless of WHO clinical stage and at } \\
\text { any CD4 cell count }\end{array}$ \\
\hline Diabetes & $\begin{array}{l}\text { Plasma fasting glucose or } \\
\text { HbA1c (where available) } \\
\text { measurements }\end{array}$ & $\begin{array}{l}\text { Oral hypoglycaemic agents; monitoring } \\
\text { glycaemic control; dietary advice; } \\
\text { promoting physical activity; physical health } \\
\text { checks and appropriate referrals; optimal } \\
\text { hypertension control; smoking cessation } \\
\text { support; aspirin; statins; and other health } \\
\text { risk modifications. Referral to diabetes care } \\
\text { after intensive phase of anti-TB treatment. }\end{array}$ \\
\hline $\begin{array}{l}\text { Depression } \\
\text { disease }\end{array}$ & $\begin{array}{l}\text { Administering the Patient } \\
\text { Health Questionnaire-9 } \\
\text { (PHQ-9) and General } \\
\text { Anxiety Disorder-7 (GAD- } \\
\text { 7) }\end{array}$ & $\begin{array}{l}\text { Brief psychotherapy and where appropriate, } \\
\text { antidepressant medications. }\end{array}$ \\
\hline $\begin{array}{l}\text { Chronic } \\
\text { Pbstructive } \\
\text { Diseanary }\end{array}$ & $\begin{array}{l}\text { Spirometry: forced } \\
\text { expiratory volume in 1s } \\
\text { FEV1) as a percentage } \\
\text { vital capacity (FVC) }\end{array}$ & $\begin{array}{l}\text { Inhaled short-acting ß2 agonists; inhaled } \\
\text { steroids and/or long-acting bronchodilators; } \\
\text { smoking cessation support; support to } \\
\text { manage symptoms of shortness of breath. }\end{array}$ \\
\hline
\end{tabular}

The key obstacles to better management of TB multimorbidity include fragmented care delivery, limited access to other specialists and financial obstacles to complex health care. A joined-up approach, specifically between TB and NCD services, to detect and manage TB multimorbidity, could better serve the needs of people with TB. This step-change may offer a better, more patient-centred healthcare where individuals do not have to negotiate multiple, disparate care pathways for their healthcare needs but instead have access to timely, coordinated care which meets their complex health needs, supports them to adapt healthy behaviours and improves the outcomes that are important to them.

\section{Acknowledgement}

"This work was supported by the Medical Research Council [grant number MC_PC_MR/T037806/1 TB Multimorbidity]; 
and by the National Institute for Health Research (NIHR) (grant 17/63/130 NIHR Global Health Research Group: Improving Outcomes in Mental and Physical Multimorbidity and Developing Research Capacity (IMPACT) in South Asia at the University of York) using UK aid from the UK Government to support global health research. The views expressed in this publication are those of the author(s) and not necessarily those of the NIHR or the UK government." 


\section{REFERENCES}

1 World Health Organization. Multimorbidity. World Health Organization, 2016.

2 Pearson-Stuttard J, Ezzati M, Gregg E W. Multimorbidity-a defining challenge for health systems. The Lancet Public Health 2019; 4(12): e599-e600.

3 Reis-Santos B, Gomes T, Macedo L R, Horta B L, Riley L W, Maciel E L. Prevalence and patterns of multimorbidity among tuberculosis patients in Brazil: a cross-sectional study. Int J Equity Health 2013; 12: 61.

$4 \quad$ Kwan C K, Ernst J D. HIV and tuberculosis: a deadly human syndemic. Clin Microbiol Rev 2011; 24(2): 351-376.

$\underline{5}$ WHO | Global tuberculosis report 2018 [Epub ahead of print].

6 Barnett K, Mercer S W, Norbury M, Watt G, Wyke S, Guthrie B. Epidemiology of multimorbidity and implications for health care, research, and medical education: a cross-sectional study. Lancet 2012; 380(9836): 37-43.

7 Lange-Maia B S, Karvonen-Gutierrez C A, Kazlauskaite R, et al. Impact of Chronic Medical Condition Development on Longitudinal Physical Function from Mid- to Early Late-Life: The Study of Women's Health Across the Nation. J Gerontol A Biol Sci Med Sci 2020; 75(7): 1411-1417.

8 Williams J S, Egede L E. The Association Between Multimorbidity and Quality of Life, Health Status and Functional Disability. Am J Med Sci 2016; 352(1): 45-52.

9 de S Santos Machado V, Valadares A L R, Costa-Paiva L H, Osis M J, Sousa M H, Pinto-Neto A M. Aging, obesity, and multimorbidity in women 50 years or older: a population-based study. Menopause 2013; 20(8): 818-824.

10 Alaba O, Chola L. The social determinants of multimorbidity in South Africa. Int J Equity Health 2013; 12: 63.

11 Jani B D, Hanlon P, Nicholl B I, et al. Relationship between multimorbidity, demographic factors and mortality: findings from the UK Biobank cohort. BMC Med 2019; 17(1): 74.

12 Marais B J, Lönnroth K, Lawn S D, et al. Tuberculosis comorbidity with communicable and non-communicable diseases: integrating health services and control efforts. Lancet Infect Dis 2013; 13(5): 436-448.

13 Koyanagi A, Vancampfort D, Carvalho A F, et al. Depression comorbid with tuberculosis and its impact on health status: cross-sectional analysis of community-based data from 48 low- and middle-income countries. BMC Med 2017; 15(1): 209.

14 Aamir S, Aisha. Co-morbid anxiety and depression among pulmonary tuberculosis patients. J Coll Physicians Surg Pak 2010; 20(10): 703-704.

15 Doherty A M, Kelly J, McDonald C, O'Dywer A M, Keane J, Cooney J. A review of the interplay between tuberculosis and mental health. Gen Hosp Psychiatry 2013; 35(4): 398-406.

16 Oh K H, Choi H, Kim E J, Kim H J, Cho S I. Depression and risk of tuberculosis: a 
nationwide population-based cohort study. Int J Tuberc Lung Dis 2017; 21(7): 804-809.

17 Maes M, Kubera M, Obuchowiczwa E, Goehler L, Brzeszcz J. Depression's multiple comorbidities explained by (neuro) inflammatory and oxidative \& nitrosative stress pathways. Neuroendocrinol Lett 2011; 32(1): 7-24.

18 Ambaw F, Mayston R, Hanlon C, Medhin G, Alem A. Untreated depression and tuberculosis treatment outcomes, quality of life and disability, Ethiopia. Bull World Health Organ 2018; 96(4): 243-255.

19 Hansel N N, Wu A W, Chang B, Diette G B. Quality of life in tuberculosis: patient and provider perspectives. Qual Life Res 2004; 13(3): 639-652.

20 Courtwright A, Turner A N. Tuberculosis and stigmatization: pathways and interventions. Public Health Rep 2010; 125 Suppl 4: 34-42.

21 Bates M, Marais B J, Zumla A. Tuberculosis Comorbidity with Communicable and Noncommunicable Diseases. Cold Spring Harb Perspect Med; 5(11).

22 Ansa G A, Walley J D, Siddiqi K, Wei X. Delivering TB/HIV services in Ghana: a comparative study of service delivery models. Trans R Soc Trop Med Hyg 2014; 108: 560-567.

23 Stubbs B, Siddiqi K, Elsey H, et al. Tuberculosis and non-communicable disease multimorbidity: an analysis of the World Health Survey in 48 low- and middle-income countries. Trop Med Int Health [Epub ahead of print].

24 Duarte R, Lönnroth K, Carvalho C, et al. Tuberculosis, social determinants and comorbidities (including HIV). Pulmonology 2018; 24(2): 115-119.

25 Noubiap J J, Nansseu J R, Nyaga U F, et al. Global prevalence of diabetes in active tuberculosis: a systematic review and meta-analysis of data from 2.3 million patients with tuberculosis. The Lancet Global Health 2019; 7(4): e448-e460.

26 van Kampen S C, Wanner A, Edwards M, et al. International research and guidelines on post-tuberculosis chronic lung disorders: a systematic scoping review. BMJ Global Health 2018; 3(4): e000745.

27 Viswanathan V, Kumpatla S, Aravindalochanan V, et al. Prevalence of diabetes and pre-diabetes and associated risk factors among tuberculosis patients in India. PLoS One 2012; 7(7): e41367.

28 Baker M A, Harries A D, Jeon C Y, et al. The impact of diabetes on tuberculosis treatment outcomes: a systematic review. BMC Med 2011; 9: 81.

29 Shahdadi H, Salarzaee M, Balouchi A. Quality of life of diabetic patients with smear positive PTB in southeastern Iran: A cross-sectional study in a poor region of Iran. Indian J Tuberc 2018; 65(2): 159-163.

30 Tegegne B S, Mengesha M M, Teferra A A, Awoke M A, Habtewold T D. Association between diabetes mellitus and multi-drug-resistant tuberculosis: evidence from a systematic review and meta-analysis. Syst Rev 2018; 7(1): 161.

31 Baghaei P, Marjani M, Javanmard P, Tabarsi P, Masjedi M R. Diabetes mellitus and tuberculosis facts and controversies. J Diabetes Metab Disord 2013; 12(1): 58.

32 Ottmani S-E, Murray M B, Jeon C Y, et al. Consultation meeting on tuberculosis and 
diabetes mellitus: meeting summary and recommendations [Meeting report]. Int $\mathrm{J}$ Tuberc Lung Dis 2010; 14(12): 1513-1517.

33 Jeon C Y, Harries A D, Baker M A, et al. Bi-directional screening for tuberculosis and diabetes: a systematic review. Tropical Medicine \& International Health 2010; 15(11): 1300-1314.

34 Byrne A L, Marais B J, Mitnick C D, Lecca L, Marks G B. Tuberculosis and chronic respiratory disease: a systematic review. Int J Infect Dis 2015; 32: 138-146.

35 Inghammar M, Ekbom A, Engström G, et al. COPD and the risk of tuberculosis--a population-based cohort study. PLoS One 2010; 5(4): e10138.

36 Marais B J, Chakaya J, Swaminathan S, et al. Tackling long-term morbidity and mortality after successful tuberculosis treatment. Lancet Infect Dis 2020; 20(6): 641642.

37 Allwood B W, van der Zalm M M, Amaral A F S, et al. Post-tuberculosis lung health: perspectives from the First International Symposium. Int J Tuberc Lung Dis 2020; 24(8): 820-828.

38 MacMahon S. Multimorbidity: A priority for global health research. The Academy of Medical Sciences: London, UK [Epub ahead of print].

39 Hurst J R, Dickhaus J, Maulik P K, et al. Global Alliance for Chronic Disease researchers' statement on multimorbidity. The Lancet Global Health 2018; 6(12): e1270-e1271.

40 Toro N. Who global strategy on integrated people-centred health services (IPCHS) / Estrategia mundial en servicios de salud integrada centrado en las personas (IPCHS). International Journal of Integrated Care; 15(8).

41 Integrating Tobacco Control into Tuberculosis and HIV Responses. UNDP. https://www.undp.org/content/undp/en/home/librarypage/hiv-aids/integrating-tobaccocontrol-into-tuberculosis-and-hiv-responses.html (accessed September 26, 2019).

42 Oni T, McGrath N, BeLue R, et al. Chronic diseases and multi-morbidity--a conceptual modification to the WHO ICCC model for countries in health transition. BMC Public Health 2014; 14: 575.

43 Creswell J, Raviglione M, Ottmani S, et al. Tuberculosis and noncommunicable diseases: neglected links and missed opportunities. Eur Respir J 2011; 37(5): 1269_ 1282.

44 Ministry of Health, Family Welfare-Government of India. India TB Report $2020::$ Ministry of Health and Family Welfare. https://tbcindia.gov.in/showfile.php?lid=3538 (accessed July 4, 2020).

45 Byrne A L, Marais B J, Mitnick C D, et al. Feasibility and yield of screening for noncommunicable diseases among treated tuberculosis patients in Peru. Int $\mathrm{J}$ Tuberc Lung Dis 2018; 22(1): 86-92.

46 Who. Package of essential noncommunicable (PEN) disease interventions for primary health care in low-resource settings.

47 Organization W H, Others. mhGAP: Mental Health Gap Action Programme: scaling up care for mental, neurological and substance use disorders [Epub ahead of print]. 
48 Akksilp S, Karnkawinpong O, Wattanaamornkiat W, et al. Antiretroviral therapy during tuberculosis treatment and marked reduction in death rate of HIV-infected patients, Thailand. Emerg Infect Dis 2007; 13(7): 1001-1007.

49 Siddiqi K, Khan A, Ahmad M, et al. Action to Stop Smoking In Suspected Tuberculosis (ASSIST) in Pakistan: a Cluster Randomized, Controlled Trial. Ann Intern Med 2013; 158: $667-675$.

50 Zellweger J-P, Cattamanchi A, Sotgiu G. Tobacco and tuberculosis: could we improve tuberculosis outcomes by helping patients to stop smoking? European Respiratory Journal 2015; 45(3): 583-585.

51 Pan S-C, Ku C-C, Kao D, Ezzati M, Fang C-T, Lin H-H. Effect of diabetes on tuberculosis control in 13 countries with high tuberculosis: a modelling study. Lancet Diabetes Endocrinol 2015; 3(5): 323-330. 